\title{
Antecedente de inmunización contra influenza en pacientes con COVID-19: impacto en la mortalidad
}

\author{
Carlos Sánchez-García, ${ }^{1}$ Juan E. Salinas-Aguirre, ${ }^{2}$ Lorena Rodríguez-Muñoz, ${ }^{1}$ Raúl Rodríguez-Sánchez, ${ }^{2}$ \\ Angélica Díaz-Castaño y Roberto Bernal-Gómez ${ }^{1}$ \\ ${ }^{1}$ Comité Técnico para la Prevención, Atención y Control de COVID-19; ${ }^{S}$ Secretaría de Salud del Estado de Coahuila de Zaragoza, Departamento \\ de Epidemiología. Coahuila, México
}

\section{Resumen}

Introducción: En el contexto de la pandemia de COVID-19 existe inquietud en cuanto al impacto de la temporada de influenza. Objetivo: Analizar el impacto del antecedente de inmunización contra influenza en pacientes con infección por SARSCoV-2. Métodos: Se incluyeron pacientes mayores de 18 años con COVID-19, registrados entre marzo y agosto de 2020. Los datos fueron analizados mediante las pruebas exacta de Fisher y $t$ de Student. Para evaluar el impacto en la mortalidad se utilizó un modelo de regresión logística; la relación entre el porcentaje de pacientes a quienes se aplicó la vacuna contra la influenza y la mortalidad fue determinada con el coeficiente de correlación de Pearson. Resultados: Se incluyeron 16879 participantes; $17 \%$ tuvo antecedente de vacunación contra influenza. La mortalidad fue menor en el grupo con historia de vacunación (3.5\% versus $7 \%, p<0.0001)$. El porcentaje de vacunación presentó una relación inversa con el porcentaje de mortalidad ( $r$ de Pearson -0.922, $p=0.026$ ). Conclusiones: La inmunización contra la influenza fue un factor protector independiente de mortalidad en pacientes con COVID-19. Aunque son necesarios más estudios para determinar la relación causal, será razonable incrementar la inmunización contra influenza en la población general.

PALABRAS CLAVE: SARS-CoV-2. COVID-19. Inmunización. Influenza. Mortalidad.

\section{History of influenza immunization in COVID-19 patients: impact on mortality}

\begin{abstract}
Introduction: In the context of the COVID-19 pandemic, there is concern regarding the impact of the influenza season. Objective: To analyze the impact of influenza immunization history on patients with SARS-CoV-2 infection. Methods: Patients older than 18 years with COVID-19, registered between March and August 2020, were included. Data were analyzed using Fisher's exact test and Student's t-test. To evaluate the impact on mortality, a logistic regression model was used; the relationship between the percentage of patients who received the influenza vaccine and mortality was determined with Pearson's correlation coefficient. Results: 16,879 participants were included; $17 \%$ had a history of influenza vaccination. Mortality was lower in the group with a history of vaccination ( $3.5 \%$ vs. $7 \%, p<0.0001)$. The vaccination rate had an inverse relationship with the mortality rate (Pearson's $r:-0.922, p=0.026)$. Conclusions: Previous influenza immunization was an independent protective factor for mortality in patients with COVID-19. Although further studies are needed to determine a causal relationship, it would be reasonable to increase influenza immunization in the general population.
\end{abstract}

KEY WORDS: SARS-COV-2. COVID-19. Immunization. Influenza. Mortality.

0016-3813/@ 2020 Academia Nacional de Medicina de México, A.C. Publicado por Permanyer. Este es un artículo open access bajo la licencia
Correspondencia:

Carlos Sánchez-García

E-mail: sanchez.carlos8516@gmail.com CC BY-NC-ND (http://creativecommons.org/licenses/by-nc-nd/4.0/).

Fecha de recepción: 09-09-2020

Fecha de aceptación: 17-09-2020 


\section{Introducción}

En diciembre de 2019 se notificó la presencia de casos de neumonía de causa desconocida en la ciudad de Wuhan, China; posteriormente, las autoridades identificaron un nuevo coronavirus (SARS-CoV-2) como el agente causal de la enfermedad (COVID-19). El 11 de marzo de 2020, la infección por SARS-CoV-2 fue declarada una pandemia y el 27 de febrero de 2020 se reportó el primer caso en México. ${ }^{1}$

Conforme la pandemia de COVID-19 ha evolucionado, se suscitó la inquietud en cuanto al impacto que podría tener la temporada de influenza. ${ }^{2}$ La influenza es una causa significativa de morbimortalidad y de uso de los servicios de salud alrededor del mundo; la segunda ola de la pandemia ha coincidido con la temporada de influenza, motivo de preocupación entre las autoridades de salud en los diferentes países. ${ }^{3,4}$

El objetivo de este estudio fue analizar el impacto del antecedente de inmunización contra influenza en pacientes con COVID-19, con la finalidad de implementar medidas que puedan impactar en la severidad de esta enfermedad.

\section{Métodos}

El diseño del estudio es una cohorte retrospectiva. Los datos fueron obtenidos del Sistema de Vigilancia de Enfermedades Respiratorias, incluido en el Sistema Nacional de Vigilancia Epidemiológica de la Dirección General de Epidemiologia, Secretaría de Salud de México. El Comité de Ética en Investigación aprobó la realización del protocolo; no se incluyeron datos que pudieran identificar individualmente a cada participante.

Se incluyeron pacientes del estado de Coahuila de Zaragoza, México, mayores de 18 años, con un resultado positivo de infección por SARS-CoV-2 obtenido mediante reacción en cadena de la polimerasa en tiempo real, registrados ente el 17 de marzo y el 16 de agosto de 2020.

Para el análisis estadístico se consideraron las siguientes variables: edad, sexo, tratamiento intrahospitalario, necesidad de ventilación mecánica, comorbilidades asociadas (diabetes mellitus, hipertensión arterial sistémica, enfermedad cardiovascular, obesidad y enfermedad renal crónica), antecedente de inmunización contra influenza durante la temporada de vacunación previa al periodo estudiado y mortalidad.
Los datos cuantitativos fueron expresados con media y desviación estándar y los datos cualitativos mediante frecuencia y porcentaje. Para la comparación entre grupos se utilizaron las pruebas exacta de Fisher y $t$ de Student independiente.

El impacto del antecedente de inmunización contra influenza en la mortalidad fue evaluado mediante el modelo de regresión logística univariado y multivariado. En la regresión logística univariada, todas las covariables fueron analizadas de forma individual; aquellas con resultado estadísticamente significativo fueron incluidas en el análisis multivariado, ajustado para la edad y el sexo.

La diferencia en el porcentaje de mortalidad entre los diferentes grupos de edad, de acuerdo con el antecedente o no de inmunización contra influenza, fue analizado mediante la prueba exacta de Fisher; asimismo, la relación entre el porcentaje de pacientes vacunados y la mortalidad entre los diferentes grupos etarios fue determinada con el coeficiente de correlación de Pearson. Un valor de $p<0.05$ fue considerado estadísticamente significativo. Para el análisis de los datos se utilizó el programa SPSS Statistics versión 21.0 para Windows (IBM Corp., Armonk, NY, Estados Unidos).

\section{Resultados}

Se incluyeron 16879 participantes, 8487 fueron del sexo femenino (50.3\%) y 8392 del masculino (49.7\%), con una media de edad de 43 años; $13.8 \%$ requirió tratamiento intrahospitalario y $17 \%$ tuvo antecedente positivo de vacunación contra influenza. La mortalidad general fue de $6.4 \%$ (1081 pacientes).

La hipertensión arterial sistémica, obesidad, diabetes mellitus, enfermedad renal crónica y enfermedad cardiovascular estuvieron presentes en 13.9, 11.9, $10.9,1.4$ y $1.3 \%$ de los pacientes, respectivamente.

Los participantes fueron divididos de acuerdo con el antecedente positivo o negativo de inmunización contra influenza; la media de edad fue discretamente mayor en el grupo sin antecedente de vacunación (43.3 años versus 41.6 años), con un valor de $p<0.0001$.

La proporción de pacientes con más de 60 años fue mayor en el grupo sin antecedente de vacunación $(p<0.0001)$. El porcentaje de participantes con diabetes mellitus $(p=0.005)$, hipertensión arterial sistémica $(p<0.001)$ y obesidad $(p<0.001)$ fue mayor en el grupo con antecedente de inmunización contra influenza.

En el grupo sin antecedente de vacunación, $14.8 \%$ requirió tratamiento intrahospitalario y $0.9 \%$ ventilación mecánica, a diferencia de 9.2 y $0.3 \%$ en el 
Tabla 1. Características de los pacientes con COVID-19 de acuerdo con el antecedente de inmunización contra influenza

\begin{tabular}{|c|c|c|c|c|c|}
\hline Característica & \multicolumn{2}{|c|}{$\begin{array}{c}\text { Con } \\
\text { inmunización } \\
(n=2905)\end{array}$} & \multicolumn{2}{|c|}{$\begin{array}{c}\text { Sin } \\
\text { inmunización } \\
(n=13974)\end{array}$} & p \\
\hline \multirow{2}{*}{$\begin{array}{l}\text { Edad en años } \\
\text { (media } \pm \text { DE) }\end{array}$} & \multicolumn{2}{|c|}{$41.6 \pm 13.9$} & \multicolumn{2}{|c|}{$43.3 \pm 15.3$} & \multirow[t]{2}{*}{$<0.0001$} \\
\hline & $\mathrm{n}$ & $\%$ & $\mathrm{n}$ & $\%$ & \\
\hline $\begin{array}{l}\text { Grupo de edad } \\
18-29 \\
30-39 \\
40-49 \\
50-59 \\
>60\end{array}$ & $\begin{array}{l}634 \\
803 \\
687 \\
456 \\
325\end{array}$ & $\begin{array}{l}21.8 \\
27.6 \\
23.6 \\
15.7 \\
11.2\end{array}$ & $\begin{array}{l}2944 \\
3301 \\
3202 \\
2402 \\
2125\end{array}$ & $\begin{array}{l}21.1 \\
23.6 \\
22.9 \\
17.2 \\
15.2\end{array}$ & $\begin{array}{c}0.369 \\
<0.0001 \\
0.397 \\
0.054 \\
<0.0001\end{array}$ \\
\hline Sexo femenino & 1328 & 45.7 & 7159 & 51.2 & $<0.0001$ \\
\hline Diabetes mellitus & 372 & 12.8 & 1529 & 11 & 0.005 \\
\hline Hipertensión arterial & 503 & 17.3 & 1845 & 13.3 & $<0.0001$ \\
\hline $\begin{array}{l}\text { Enfermedad } \\
\text { cardiovascular }\end{array}$ & 37 & 1.3 & 174 & 1.3 & 0.935 \\
\hline Obesidad & 473 & 16.3 & 1531 & 11 & $<0.0001$ \\
\hline $\begin{array}{l}\text { Enfermedad renal } \\
\text { crónica }\end{array}$ & 42 & 1.4 & 208 & 1.5 & 0.926 \\
\hline $\begin{array}{l}\text { Tratamiento } \\
\text { intrahospitalario }\end{array}$ & 266 & 9.2 & 2067 & 14.8 & $<0.0001$ \\
\hline Ventilación mecánica & 8 & 0.3 & 122 & 0.9 & 0.001 \\
\hline Mortalidad & 101 & 3.5 & 980 & 7 & $<0.0001$ \\
\hline
\end{tabular}

grupo con antecedente. La mortalidad fue significativamente menor en el grupo con historia positiva de vacunación contra influenza (3.5\% versus $7 \%$ ), con un valor de $p<0.0001$. El resto de las características de los pacientes se presentan en la Tabla 1.

Mediante regresión logística univariada se observó que la edad mayor de 60 años, diabetes mellitus, hipertensión arterial sistémica, enfermedad cardiovascular, obesidad y enfermedad renal crónica se asociaron a incremento en la mortalidad en pacientes con COVID-19 ( $p$ < 0.0001). El antecedente de vacunación contra influenza resultó ser un factor protector para mortalidad, en el que se demostró razón de momios $(\mathrm{RM})=0.47$ con un intervalo de confianza de $95 \%($ IC $95 \%)=0.38-0.58$.

Estos datos fueron analizados conjuntamente mediante un modelo de regresión logística multivariado, ajustado para la edad y el sexo. Aunque la mayoría de las covariables demostraron ser factores de riesgo independientes para mortalidad, el antecedente de vacunación contra influenza presentó $\mathrm{RM}=0.44$, IC
$95 \%=0.35-0.55$, con un valor de $p<0.0001$, por lo que constituyó un factor protector independiente contra mortalidad en pacientes con COVID-19 (Figura 1).

La diferencia en el porcentaje de mortalidad entre los participantes con y sin antecedente de vacunación de acuerdo con cada grupo etario demostró ser superior al incrementarse la edad; fue mayor en los participantes de más de 60 años $(p=0.003)$ (Figura 2).

Entre los diferentes grupos de edad, el porcentaje de vacunación presentó una relación inversa con el porcentaje de mortalidad (coeficiente de correlación de Pearson -0.922), con un valor de $p=0.026$ (Figura 3).

\section{Discusión}

Se han observado diferentes grados de susceptibilidad a la infección por SARS-CoV-2 entre los diferentes grupos de edad, diferencias que podrían estar determinadas por la disparidad en la respuesta inmune (influida por el antecedente de infecciones e inmunizaciones previas). ${ }^{5}$ Existen algunos estudios que evalúan la relación entre el antecedente de vacunación contra influenza y la disminución de la severidad de COVID-19. ${ }^{6}$

En Italia se reportó que la vacuna antineumocócica y antiinfluenza podrían estar asociadas a menor probabilidad de infección por SARS-CoV-2. ${ }^{7}$ En otro estudio se encontró una correlación negativa entre el porcentaje de muertes por COVID-19 y el porcentaje de adultos mayores de 65 años con inmunización contra influenza. ${ }^{8}$ En nuestro protocolo encontramos esta correlación, sin embargo, analizamos el porcentaje de vacunación solo de los pacientes con COVID19 y no de la población general, como sucedió en ambos estudios italianos.

En dos reportes, los cuales aún no han sido sometidos a revisión por pares, en Estados Unidos de América (Zanettini et al., 2020) y en Brasil (Fink et al., 2020), se sugiere un potencial efecto protector de la vacuna contra influenza respecto a la mortalidad en pacientes con COVID-19..$^{9,10}$

Nosotros encontramos que al parecer el antecedente de inmunización contra influenza constituye un efecto protector independiente respecto a la mortalidad por COVID-19; aparentemente, la diferencia es mayor en los pacientes de más de 60 años, por lo que concordamos con los estudios comentados.

Hasta donde conocemos, este es el primer estudio en México y uno de los pocos en Latinoamérica que analiza el impacto del antecedente de vacunación contra influenza en pacientes con COVID-19. 


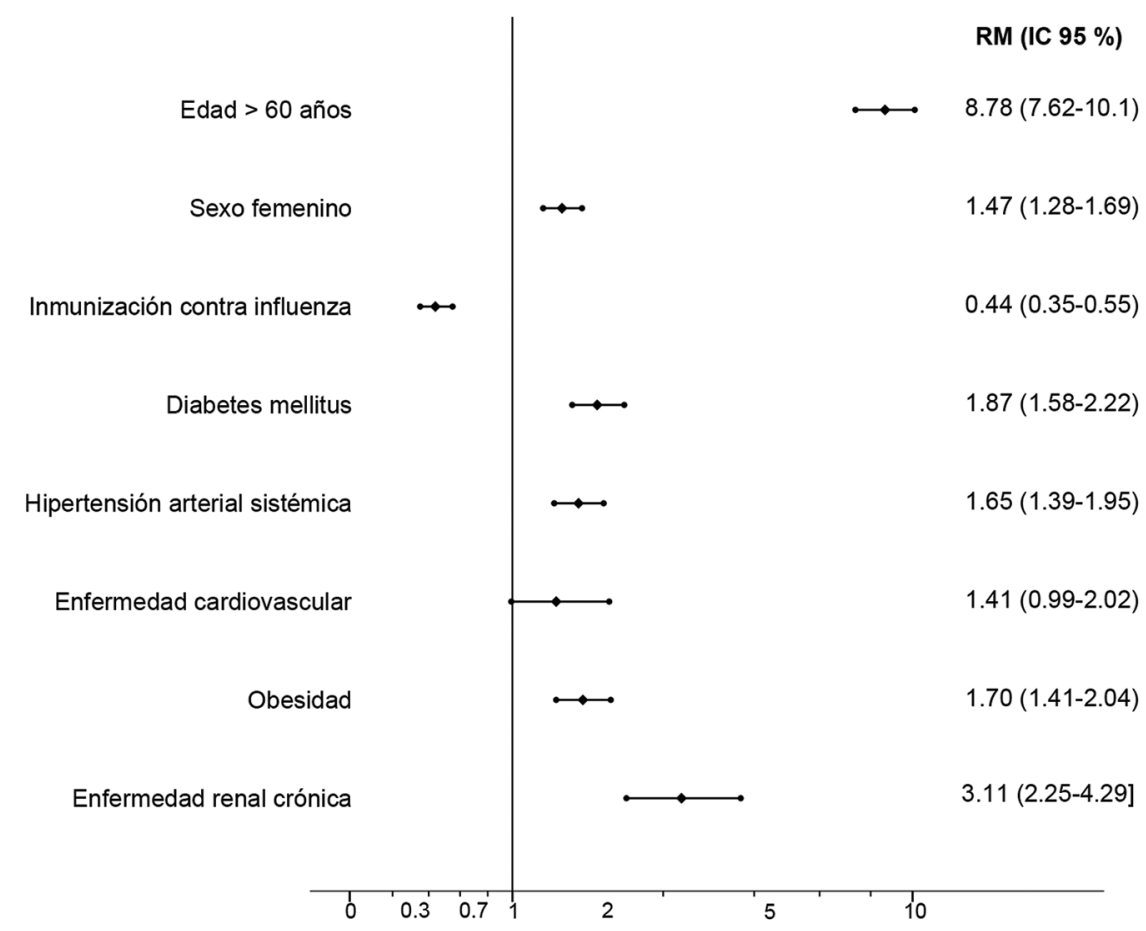

Figura 1. Análisis multivariado de los factores relacionados con mortalidad en pacientes con COVID-19. RM = razón de momios, IC $95 \%=$ intervalo de confianza de $95 \%$.

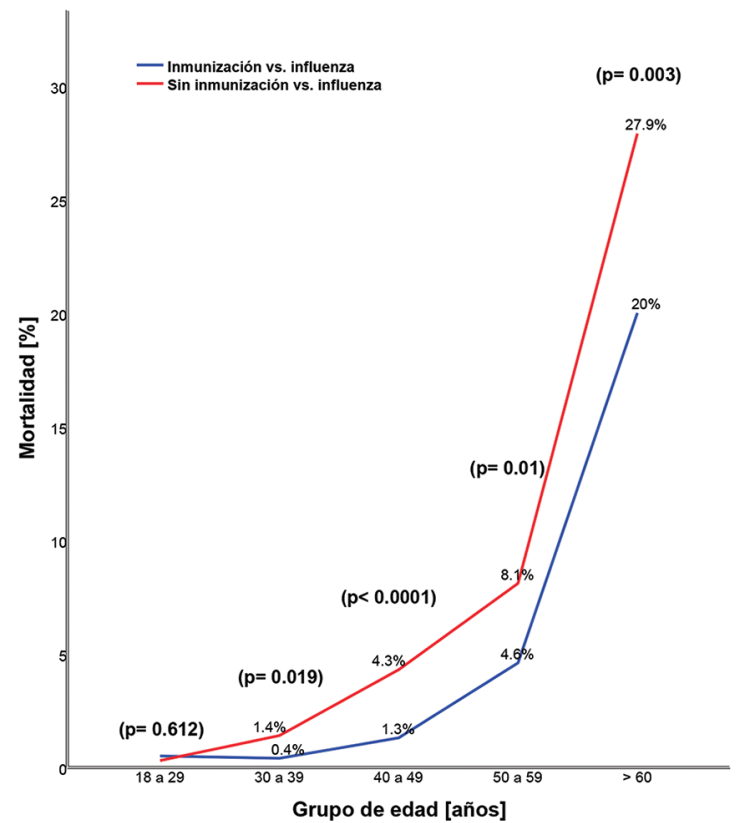

Figura 2. Comparación entre el porcentaje de mortalidad de pacientes con y sin antecedente de inmunización contra influenza, de acuerdo con el grupo de edad.

Aun cuando algunos estudios han abordado el posible efecto protector de la inmunización contra influenza en la mortalidad por COVID-19, otros factores podrían explicar estos hallazgos.

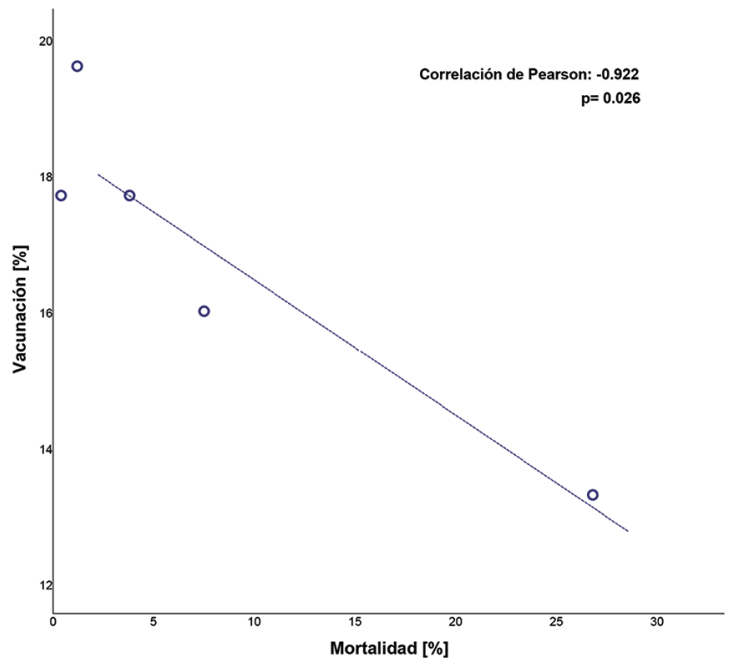

Figura 3. Correlación entre el porcentaje de pacientes vacunados y el porcentaje de mortalidad en pacientes con infección por SARS-CoV-2.

La inmunización contra influenza podría, al menos en parte, fomentar inmunidad contra SARS-CoV-2, debido al estímulo inmunológico generado por esta vacuna. ${ }^{11}$ Se han reportado altos índices de coinfección por SARS-CoV-2 y virus de la influenza. Ahora bien, el acceso a la vacuna contra influenza puede estar relacionado con un estrato socioeconómico 
mayor, lo que estaría relacionado con acceso a mejores servicios de salud. ${ }^{12-14}$ Todos estos factores podrían contribuir a la asociación entre inmunización contra influenza y la infección por SARS-CoV-2 y el aparente factor protector podría no estar directamente relacionado.

Reconocemos las limitantes de nuestro estudio, como su naturaleza retrospectiva y que el método de obtención de los datos podría estar asociado a sesgos.

En conclusión, el antecedente de inmunización contra influenza parece ser un factor protector independiente de mortalidad en pacientes con COVID-19; sin embargo, este resultado podría ser producto de la casualidad o de los factores comentados. No obstante, es de gran interés ya que podría ser un factor modificable que impactaría en la severidad de la infección por SARS-CoV-2, de ahí la necesidad de más estudios para determinar si existe alguna relación causal. A pesar de lo anterior, es razonable realizar esfuerzos para incrementar el porcentaje de inmunización contra la influenza en la población en el contexto de la pandemia de COVID-19.

\section{Conflicto de intereses}

Los autores declaran no tener conflictos de intereses alguno.

\section{Financiamiento}

Los autores no recibieron patrocinio para llevar a cabo este artículo.

\section{Responsabilidades éticas}

Protección de personas y animales. Los autores declaran que para esta investigación no se realizaron experimentos en seres humanos ni en animales.
Confidencialidad de los datos. Los autores declaran que siguieron los protocolos de su centro de trabajo sobre la publicación de datos de pacientes.

Derecho a la privacidad y consentimiento informado. Los autores declaran que en este artículo no aparecen datos de pacientes.

\section{Bibliografía}

1. Suárez V, Suárez-Quezada M, Oros-Ruiz S, Ronquillo-de Jesús E. Epidemiología de COVID-19 en México: del 27 de febrero al 30 de abril de 2020. Rev Clin Esp. 2020;8:463-471.

2. Singer BD. COVID-19 and the next influenza season. Sci Adv. 2020;6:eabd0086.

3. Grech V, Borg M. Influenza vaccination in the COVID-19 era. Early Hum Dev. 2020;148:105116.

4. Maltezou HC, Theodoridou K, Poland G. Influenza immunization and COVID-19. Vaccine. 2020;38:6078-6079.

5. Salem ML, El-Hennawy D. The possible beneficial adjuvant effect of influenza vaccine to minimize the severity of COVID-19. Med Hypotheses. 2020;140:109752.

6. Osaras R, Cirpin R, Duman H, Duran A, Arslan O, Leblebicioglu H. An open call for influenza vaccination pending the new wave of COVID-19. J Med Virol. 2020

7. Noale M, Trevisan C, Maggi S, Antonelli-Incalzi R, Pedone C, Di Bari M, et al. The association between influenza and pneumococcal vaccinations and SAS-CoV-2 Infection: data from the EPICOVID19 Web-Based Survey. Vaccines (Basel). 2020;8:471

8. Marín-Hernández D, Schwartz RE, Nixon DF. Epidemiological evidence for association between higher influenza vaccine uptake in the elderly and lower COVID-19 deaths in Italy. J Med Virol. 2020;10.1002/ jmv.26120.

9. Zanettini C, Omar M, Dinalankara W, Imana EL, Colantuoni E, Parmigiani $\mathrm{G}$, et al. Influenza vaccination and COVID-19 mortality in the USA. medRxiv. Preprint. 2020 Jun 26.

10. Fink G, Orlova-Fink N, Schindler T, Grisi S, Ferrer AP, Daubenberger C, et al. Inactivated trivalent influenza vaccine is associated with lower mortality among Covid-19 patients in Brazil. medRxiv. Preprint. 2020. Dec 11.

11. Zheng J, Perlman S. Immune responses in influenza A virus and human coronavirus infection: an ongoing battle between the virus and host. Curr Opin Virol. 2017;28:43-52.

12. Yue H, Zhang M, Xing L, Wang K, Rao X, Liu H, et al. The epidemiology and clinical characteristics of co-infection of SARS-CoV-2 and influenza viruses in patients during COVID-19 outbreak. J Med Virol. 2020;92:2870-2873.

13. Lucyk K, Simmonds KA, Lorenzetti DL, Drews SJ, Svenson LW, RusseII ML. The association between influenza vaccination and socioeconomic status in high income countries varies by measure used: a systematic review. BMC Med Res Methodol.. 2019;19:153.

14. Okoli GN, Abou-Setta AM, Neilson CJ, Chit A, Thommes E, Mahmud SM. Determinants of seasonal influenza vaccine uptake among the elderly in the United States: a systematic review and meta-analysis. Gerontol Geriatr Med. 2019:5:2333721419870345. 